\title{
Fourth ventricle tumors in children: complications and influence of surgical approach
}

\author{
Sebastian M. Toescu, MBChB(Hons), MRCS,1,2 Gargi Samarth, MBBS,1 \\ Hugo Layard Horsfall, MBBS, ${ }^{1,3}$ Richard Issitt, DClinP, ${ }^{4}$ Ben Margetts, PhD, ${ }^{4}$ \\ Kim P. Phipps, BSc(Hons), ${ }^{1}$ Noor-ul-Owase Jeelani, MBA, MPhil (Medical Law), FRCS(SN), ${ }^{1}$ \\ Dominic N. P. Thompson, MBBS, FRCS(SN), ${ }^{1}$ and Kristian Aquilina, MD, FRCS(SN) ${ }^{1}$
}

\begin{abstract}
1Department of Neurosurgery, Great Ormond Street Hospital for Children, London; ${ }^{2}$ Developmental Imaging and Biophysics Section, UCL GOS Institute of Child Health, London; ' ${ }^{2}$ eppartment of Neurosurgery, Addenbrooke's Hospital, Cambridge; and ${ }^{4}$ Digital Research Environment, Great Ormond Street Hospital for Children, DRIVE Office, London, United Kingdom
\end{abstract}

\begin{abstract}
OBJECTIVES The goal of this study was to characterize the complications and morbidity related to the surgical management of pediatric fourth ventricle tumors.

METHODS All patients referred to the authors' institution with posterior fossa tumors from 2002 to 2018 inclusive were screened to include only true fourth ventricle tumors. Preoperative imaging and clinical notes were reviewed to extract data on presenting symptoms; surgical episodes, techniques, and adjuncts; tumor histology; and postoperative complications.

RESULTS Three hundred fifty-four children with posterior fossa tumors were treated during the study period; of these, 185 tumors were in the fourth ventricle, and 167 fourth ventricle tumors with full data sets were included in this analysis. One hundred patients were male (mean age $\pm \mathrm{SD}, 5.98 \pm 4.12$ years). The most common presenting symptom was vomiting (63.5\%). The most common tumor types, in order, were medulloblastoma (94 cases) > pilocytic astrocytoma (30 cases) > ependymoma (30 cases) > choroid plexus neoplasms (5 cases) > atypical teratoid/rhabdoid tumor (4 cases), with 4 miscellaneous lesions. Of the $67.1 \%$ of patients who presented with hydrocephalus, $45.5 \%$ had an external ventricular drain inserted (66.7\% of these prior to tumor surgery, $56.9 \%$ frontal); these patients were more likely to undergo ventriculoperitoneal shunt (VPS) placement at a later date $(p=0.00673)$. Twenty-two had an endoscopic third ventriculostomy, of whom 8 later underwent VPS placement. Overall, $19.7 \%$ of patients had a VPS sited during treatment.

Across the whole series, the transvermian approach was more frequent than the telovelar approach (64.1\% vs $33.0 \%)$; however, the telovelar approach was significantly more common in the latter half of the series $(p<0.001)$. Gross-total resection was achieved in $70.7 \%$. The most common postoperative deficit was cerebellar mutism syndrome (CMS; $28.7 \%$ ), followed by new weakness $(24.0 \%)$, cranial neuropathy $(18.0 \%)$, and new gait abnormality/ataxia (12.6\%). Use of intraoperative ultrasonography significantly reduced the incidence of CMS ( $p=0.0365)$. There was no significant difference in the rate of CMS between telovelar or transvermian approaches $(p=0.745)$, and multivariate logistic regression modeling did not reveal any statistically significant relationships between CMS and surgical approach.
\end{abstract}

CONCLUSIONS Surgical management of pediatric fourth ventricle tumors continues to evolve, and resection is increasingly performed through the telovelar route. CMS is enduringly the major postoperative complication in this patient population.

https://thejns.org/doi/abs/10.3171/2020.6.PEDS2089

KEYWORDS cerebellar mutism syndrome; telovelar; complications; posterior fossa tumors; fourth ventricle; oncology; surgical technique

$\mathrm{T}$ UMORS of the fourth ventricle in children pose a particular surgical challenge due to the proximity of eloquent neural parenchyma in the region. The roof and walls of the fourth ventricle contain key elements of the efferent cerebellar pathway-the dentate nuclei and superior cerebellar peduncle-and the floor of the fourth ventricle, formed by the brainstem, contains several cranial nerve nuclei on its surface. Surgical access to tumors occupying or invading the fourth ventricle has, since the time of Cushing in the 1920s, ${ }^{1}$ been performed through

ABBREVIATIONS CMS = cerebellar mutism syndrome; $\mathrm{CPA}=$ cerebellopontine angle; $\mathrm{CUSA}=\mathrm{Cavitron}$ ultrasonic surgical aspirator; $\mathrm{EOR}=$ extent of resection; $\mathrm{ETV}=$ endoscopic third ventriculostomy; EVD = external ventricular drain; GTR = gross-total resection; STR = subtotal resection; VPS = ventriculoperitoneal shunt.

SUBMITTED February 11, 2020. ACCEPTED June 10, 2020.

INCLUDE WHEN CITING Published online October 23, 2020; DOI: 10.3171/2020.6.PEDS2089. 
a direct transvermian route. The telovelar approach, first described in $1992,{ }^{2}$ makes use of the natural corridor from the cisterna magna to the fourth ventricle along the cerebellomedullary fissure, and thus spares transgression of the cerebellar vermis. This has been thought to influence the development of postoperative truncal ataxia and cerebellar mutism syndrome (CMS). ${ }^{3}$ Reports of neurological morbidity following fourth ventricle tumor resection are largely restricted to small pediatric ${ }^{4-9}$ or combined adult and pediatric series, ${ }^{10-12}$ and describe patient groups that are heterogeneous with respect to preoperative comorbidities, tumor location within the posterior fossa, and tumor histology and grade.

The objective of this retrospective single-center series of consecutive cases was to critically assess early, surgeryrelated morbidity in pediatric fourth ventricle tumors over 2 decades, with particular reference to surgical approach.

\section{Methods}

This study was registered under local research and development protocols, and ethical approval was granted from the institutional review board. Prospectively maintained dedicated neuro-oncology databases were retrospectively interrogated to identify all children referred to our academic tertiary referral center with a new posterior fossa tumor between January 1, 2002, and December 31, 2018, inclusive. Preoperative axial imaging of these patients was reviewed by the authors (S.M.T., G.S., H.L.H., K.A.) to screen for true fourth ventricle tumors. This included tumors in the fourth ventricle with extensions into the vermis or medial cerebellar hemispheres, cerebellopontine angle (CPA) via foramina of Luschka, and exophytic midbrain or medullary tumors. Tumors purely in the cerebellar hemispheres, CPA, and nonexophytic intrinsic brainstem tumors were excluded from the cohort. Tumor volumes were estimated using the $\mathrm{ABC} / 2$ method. ${ }^{13}$

Clinical notes were reviewed to provide dichotomized data points on presenting symptoms (and their duration in days); surgical episodes, techniques, and adjuncts; tumor histology; and postoperative complications. Extent of resection (EOR) was graded as gross-total resection (GTR), subtotal resection (STR), or biopsy only, by reviewing operative notes and postoperative imaging. The clinical course of patients included in the study was assessed over the first 30 postoperative days. Postoperative pediatric CMS was defined as present or absent based on the description of mutism in the contemporaneous medical notes by the attending neurosurgeon or multidisciplinary staff, who covered pediatric neurology, physiotherapy, and speech and language therapy. Postoperative weakness was defined as new postoperative focal motor weakness or long tract signs such as hypotonia or hyperreflexia. The presence of new postoperative ataxia, diplopia, and cranial nerve palsies, where these were not present preoperatively, was also noted. Further data points were collected on the presence of CSF leak, infection, hemorrhage (and subsequent evacuation), stroke, and mortality. Patients without valid data points for surgical approach were excluded from the cohort by listwise deletion.
When urgent CSF diversion was required prior to definitive tumor resection, patients underwent either insertion of an external ventricular drain (EVD) or endoscopic third ventriculostomy (ETV). Otherwise, children were temporized with glucocorticoid administration before tumor resection. Resective surgery of fourth ventricle tumors was performed by consultant neurosurgeons.

Demographic data for patients included in the cohort were extracted from our institution's clinical databases by using a direct SQL (Structured Query Language) inquiry. Data processing and analysis were carried out in $\mathrm{R}$ version 3.5.0. ${ }^{14}$ and graphics were created using the ggplot 2 package.$^{15}$ To test for differences in proportions of categorical variables, the chi-square test was used, or Fisher's exact test was used where numbers were small or proportions extreme, with an a priori $\alpha$ level of 0.05 . The Shapiro-Wilk test was used to check normality of continuous variables prior to hypothesis testing with either a t-test or Wilcoxon signed-rank test. Bonferroni multiple comparison correction was used within families of tests, represented in this paper as separate tables. Multivariate logistic regression models were built to explore relationships between binary explanatory and response variables.

\section{Results}

\section{Presentation and Demographics}

Three hundred fifty-four children with posterior fossa tumors were seen during the study period (20.8/year). After screening of preoperative imaging, 185 fourth ventricle tumors were identified; 167 fourth ventricle tumors with full data sets were included in this analysis (Fig. 1). The mean age at presentation was 5.98 years (SD 4.12 years); 100 patients were male (59.9\%). Hydrocephalus was seen in 112 patients (67.1\%) at presentation. The most common presenting symptom was vomiting $(106 / 167,63.5 \%)$, followed by ataxia $(86 / 167,51.5 \%)$, headache $(81 / 167,48.5 \%)$, visual change $(57 / 167,34.1 \%)$, long tract signs $(31 / 167,18.6 \%)$, and cranial neuropathy $(27 / 167,16.2 \%)$. Symptoms were present for a median of 28 days prior to admission (range 1-270 days). Differences were observed in the frequency of ages at which different symptoms occurred (Fig. 2 left). The average age of children presenting with ataxia was significantly younger $(p=0.0384)$, whereas visual change $(p<0.001)$ and headache $(\mathrm{p}<0.001)$ were significantly more likely to be present in older children. Clustering analysis (Fig. 2 right) revealed that symptoms of raised intracranial pressure (vomiting and headache) and more "global" neurological phenomena such as ataxia and hydrocephalus tended to group together. A separate cluster was observed for more focal symptoms, such as cranial neuropathy and visual change.

\section{Hydrocephalus Management}

Figure 1 depicts the management of children with hydrocephalus. Fifty-one patients had an EVD inserted; 34 of these devices were sited prior to definitive tumor resection, 9 were sited during the operation to resect the tumor (with 9 more having a transparietal ventricular tap performed intraoperatively), and 8 were sited after tumor re- 


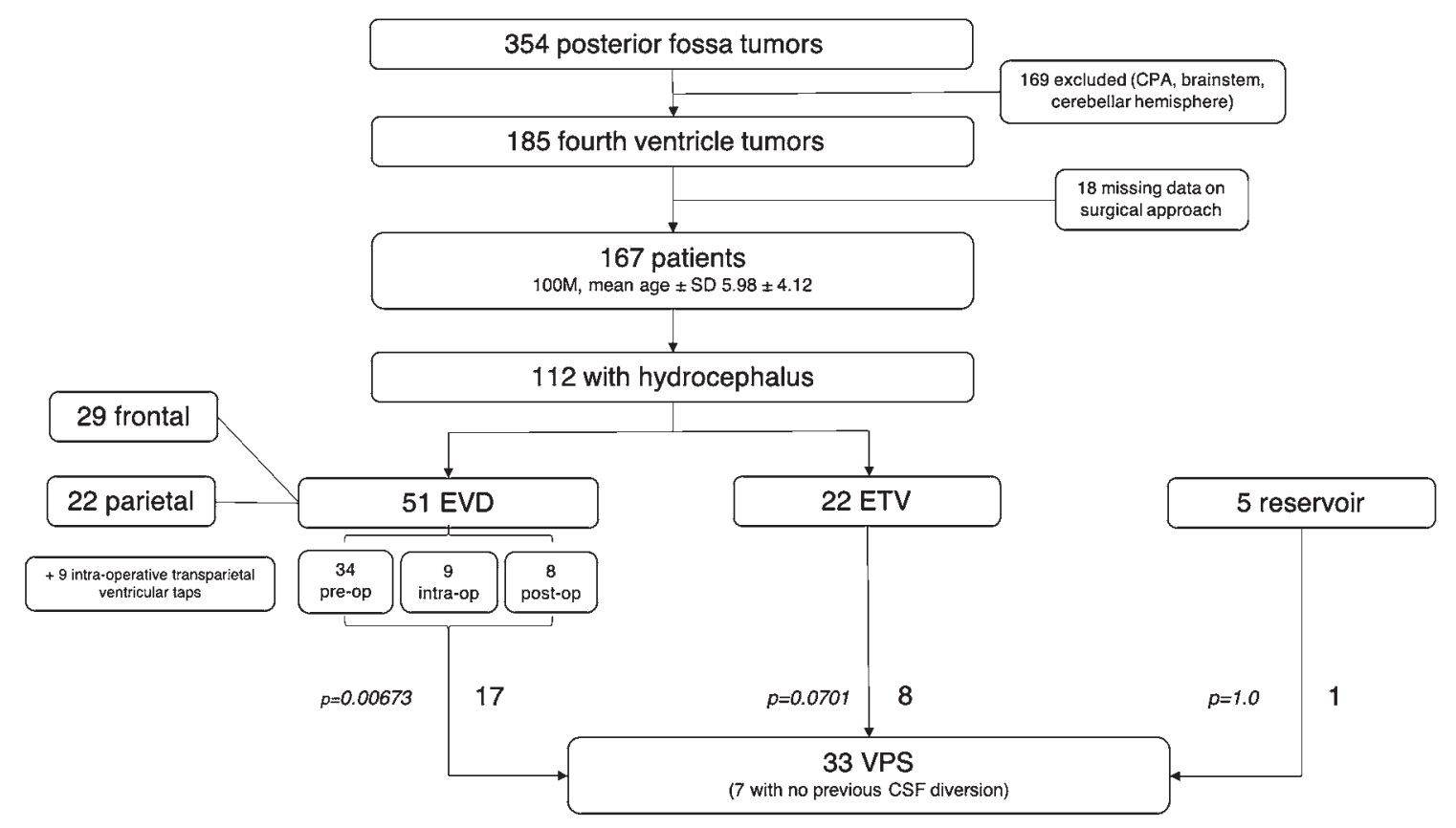

FIG. 1. Flowchart of patient inclusion and hydrocephalus management. The p values are derived from chi-square or Fisher's exact tests; see Hydrocephalus Management for details.
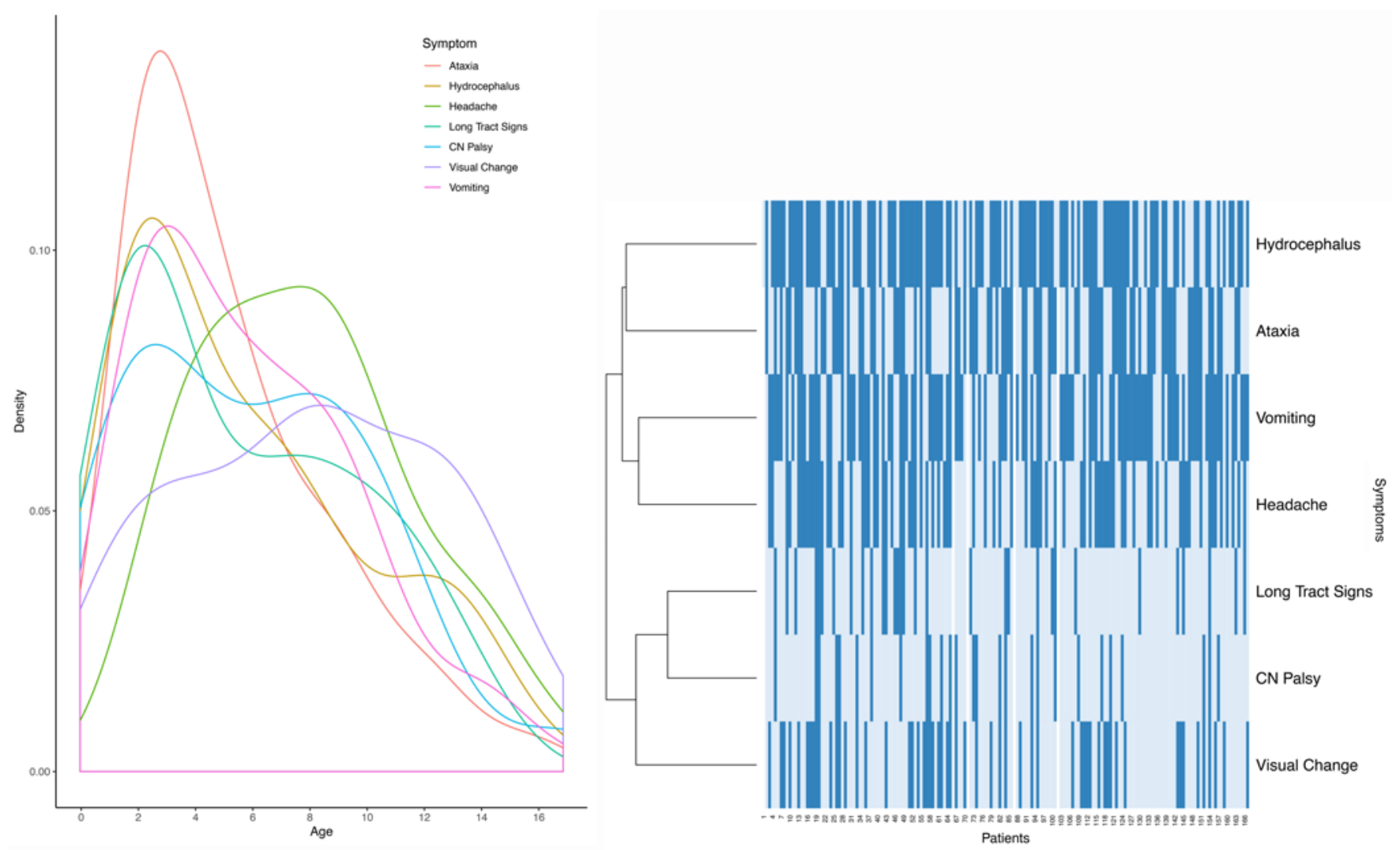

FIG. 2. Left: Density plot showing frequency of age at presentation for different symptoms. Right: Heat map showing clustering analysis of symptoms. Each column represents 1 patient, each row 1 symptom; darker bars indicate where a symptom was present. Linked lines on the left of the plot indicate clusters of symptoms that tended to group together in an individual patient. $\mathrm{CN}=$ cranial nerve. 
TABLE 1. Tumor histology and postoperative morbidity in 167 patients with fourth ventricle tumors

\begin{tabular}{lcc}
\hline \multicolumn{1}{c}{ Characteristic } & No. & $\%$ \\
\hline Histology & & \\
\hline Medulloblastoma & 94 & 56.3 \\
\hline Ependymoma & 30 & 18.0 \\
\hline Pilocytic astrocytoma & 30 & 18.0 \\
\hline Choroid plexus neoplasm & 5 & 2.99 \\
\hline ATRT & 4 & 2.40 \\
\hline Other & 4 & 2.40 \\
\hline Postop morbidity & & \\
\hline CMS & 48 & 28.7 \\
\hline Weakness & 40 & 24.0 \\
\hline Cranial neuropathy & 30 & 18.0 \\
\hline Infection & 21 & 12.6 \\
\hline Gait abnormality & 21 & 12.6 \\
\hline Diplopia & 14 & 8.38 \\
\hline CSF leak & 7 & 4.19 \\
\hline Hemorrhage & 5 & 2.99 \\
\hline Sensory deficit & 4 & 2.40 \\
\hline 30-day mortality & 4 & 2.40 \\
\hline Evacuation of hemorrhage & 0 & 0 \\
\hline Stroke & 0 & 0 \\
\hline
\end{tabular}

ATRT = atypical teratoid/rhabdoid tumor.

section, due to either pseudomeningocele or late postoperative hydrocephalus. Twenty-nine EVDs were transfrontal and 22 were transparietal. There was a significantly higher proportion of patients who underwent eventual ventriculoperitoneal shunt (VPS) insertion after EVD placement $(17 / 51,33.3 \%)$ compared with those who did not have a prior EVD $(16 / 116,13.8 \%$; difference in proportions $19.5 \%, 95 \%$ CI $6.0 \%-34.2 \%, p=0.00673$ ). Eight of 22 patients (36.4\%) undergoing ETV later underwent VPS insertion, although there was no statistically significant difference in the proportion receiving a shunt compared with those who had not had an ETV $(25 / 145,17.2 \%$; difference in proportions $19.1 \%, 95 \%$ CI $1.1 \%-40.5 \%, \mathrm{p}=0.0701)$. Five patients (all aged $<1$ year) had a CSF reservoir placed during treatment, with one undergoing eventual VPS insertion (Fisher's test, $\mathrm{p}=1.0)$. Overall, 33 patients $(19.8 \%)$ underwent VPS placement during this series; 8 were de novo, with the remainder having undergone prior CSF diversionary procedures.

\section{Surgical Techniques}

Eighty-one of 167 patients (48.5\%) were placed in the sitting position; 86/167 patients (51.5\%) were positioned prone. The cohort was divided into two broadly equal epochs: early (2009 or earlier, $n=84)$ and late (2010 or later, $\mathrm{n}=83$ ). In the early cohort, the sitting position was used in 57/84 cases (67.9\%); in the late epoch, this figure was 24/83 (28.9\%; difference in proportions $38.9,95 \%$ CI $24.0 \%-51.4 \%, \mathrm{p}<0.001)$. The posterior fossa was exposed via a suboccipital craniotomy in all but one case, in which a craniectomy was undertaken. The intradural surgical approach to the fourth ventricle was transvermian in $107(64.1 \%)$ and telovelar in $55(33.0 \%)$ patients. Five patients $(3.0 \%)$ were treated via other approaches: 3 underwent biopsy only, and in 2 the tumor was accessed directly on dural opening as it protruded through the foramen of Magendie (GTR was achieved in both of these cases). In the early epoch, the telovelar approach was used in 10/81 (12.3\%) cases; in the late epoch, this figure was 45/81 (55.6\%; difference in proportions $43.2 \%$, 95\% CI $29.2 \%-54.9 \%, p<0.001)$. Larger tumors tended to be approached by the transvermian route $\left(39.1 \mathrm{~cm}^{3}\right.$ vs $31.7 \mathrm{~cm}^{3}$ for the telovelar group), although this result did not survive multiple comparison correction. Intraoperative neuronavigation and neurophysiological monitoring were used in a minority of cases (both were used in 5/167, $3.0 \%$ ), whereas intraoperative ultrasonography was used in 49/167 (29.3\%). A Cavitron ultrasonic surgical aspirator (CUSA) was used in 98/167 (58.7\%), and fixed retractors were used in only 1 case.

EOR was graded as GTR in 117/167 (70.1\%), STR in $47 / 167(28.1 \%)$, and biopsy only in $3(1.8 \%)$ cases. Table 1 shows tumor histology; medulloblastoma was the most common $(94 / 167,56.3 \%)$. The "other" category comprised 1 embryonal tumor with abundant neuropil and true rosettes (ETANTR), 1 teratoma, 1 juvenile xanthogranuloma, and 1 high-grade glioma.

\section{Postoperative Morbidity and Mortality}

Forty-nine patients (29.3\%) had no postoperative complications documented. The remainder had at least one postoperative complication documented (Table 1). The most common was CMS, which occurred in 48/167 patients (28.7\%) overall across the cohort. Table 2 shows patient features that were explored for their association with CMS. Children who developed CMS tended to be younger (mean age 5.21 vs 6.29 years) and to have tumor volumes that were statistically significantly larger $\left(42.0 \mathrm{~cm}^{3} \mathrm{vs} 34.1\right.$ $\mathrm{cm}^{3}$ ), although this relationship did not survive multiple comparison correction. The proportion of children developing CMS was not related to whether they had presented with hydrocephalus $(p=0.741)$. The rate of CMS in any given year did not appear to be associated with utilization of different surgical approaches in that year (Fig. 3). Nor was there any association between surgical approach and CMS $(p=0.745)$ across the whole series. Multivariate logistic regression modeling including age at presentation, surgical approach, and hydrocephalus at presentation did not reveal any additional statistically significant relationships between these variables and the development of CMS.

In addition, the presence of CMS was not related to intraoperative neuronavigation use $(\mathrm{p}=0.625)$, CUSA use $(\mathrm{p}=0.482)$, or EOR $(\mathrm{p}=0.601)$. The use of intraoperative ultrasound imaging, however, was found to be protective against the development of postoperative CMS ( $p=$ 0.0365 ), although this result did not survive Bonferroni correction.

Table 3 demonstrates the association of surgical approach and all elements of postoperative neurological morbidity. Forty of 167 patients $(24.0 \%)$ had new or 
TABLE 2. Association of patient features with CMS

\begin{tabular}{|c|c|c|c|c|}
\hline Characteristic & CMS + & CMS - & Difference $(95 \% \mathrm{Cl})$ & $p$ Value \\
\hline Age at presentation, yrs & 5.21 & 6.29 & $1.08(0.41,1.75)$ & 0.121 \\
\hline Mean tumor volume, $\mathrm{cm}^{3}$ & 42.0 & 34.1 & $7.9(6.37,9.43)$ & 0.0139 \\
\hline \multicolumn{5}{|c|}{ Hydrocephalus at presentation } \\
\hline Yes & $34 / 48(70.8 \%)$ & $78 / 119(65.5 \%)$ & \multirow{2}{*}{$5.3 \%(-10.8,19.4)$} & \multirow{2}{*}{0.634} \\
\hline No & $14 / 48(29.2 \%)$ & $41 / 119(34.5 \%)$ & & \\
\hline \multicolumn{5}{|l|}{ Intraop CUSA* } \\
\hline Yes & $24 / 98(24.5 \%)$ & $18 / 58(31.0 \%)$ & \multirow{2}{*}{$6.5 \%(-7.5,21.3)$} & \multirow{2}{*}{0.482} \\
\hline No & $74 / 98(75.5 \%)$ & $40 / 58(69.0 \%)$ & & \\
\hline \multicolumn{5}{|l|}{ Intraop ultrasonography† } \\
\hline Used & $8 / 47(17.0 \%)$ & $41 / 117(35.0 \%)$ & \multirow{2}{*}{$18.0 \%(2.6,30.1)$} & \multirow{2}{*}{0.0365} \\
\hline Not used & $39 / 47(83.0 \%)$ & $76 / 117(65.0 \%)$ & & \\
\hline \multicolumn{5}{|l|}{ Intraop neuronavigation† } \\
\hline Used & $2 / 47(4.26 \%)$ & $3 / 117(2.56 \%)$ & \multirow{2}{*}{$1.7 \%(-3.9,11.8)$} & \multirow{2}{*}{0.625} \\
\hline Not used & $45 / 47(95.7 \%)$ & $114 / 117(97.4 \%)$ & & \\
\hline \multicolumn{5}{|l|}{ EOR } \\
\hline GTR & $35 / 48(72.9 \%)$ & $83 / 119(69.7 \%)$ & \multirow{3}{*}{ NA } & \multirow{3}{*}{0.601} \\
\hline STR & $12 / 48(25.0 \%)$ & $34 / 119(28.6 \%)$ & & \\
\hline Biopsy & $1 / 48(2.08 \%)$ & $2 / 119(1.68 \%)$ & & \\
\hline
\end{tabular}

NA = not applicable.

* No data for 11 patients.

† No data for 3 patients. Bonferroni significance level 0.05/7 $=0.00714$.

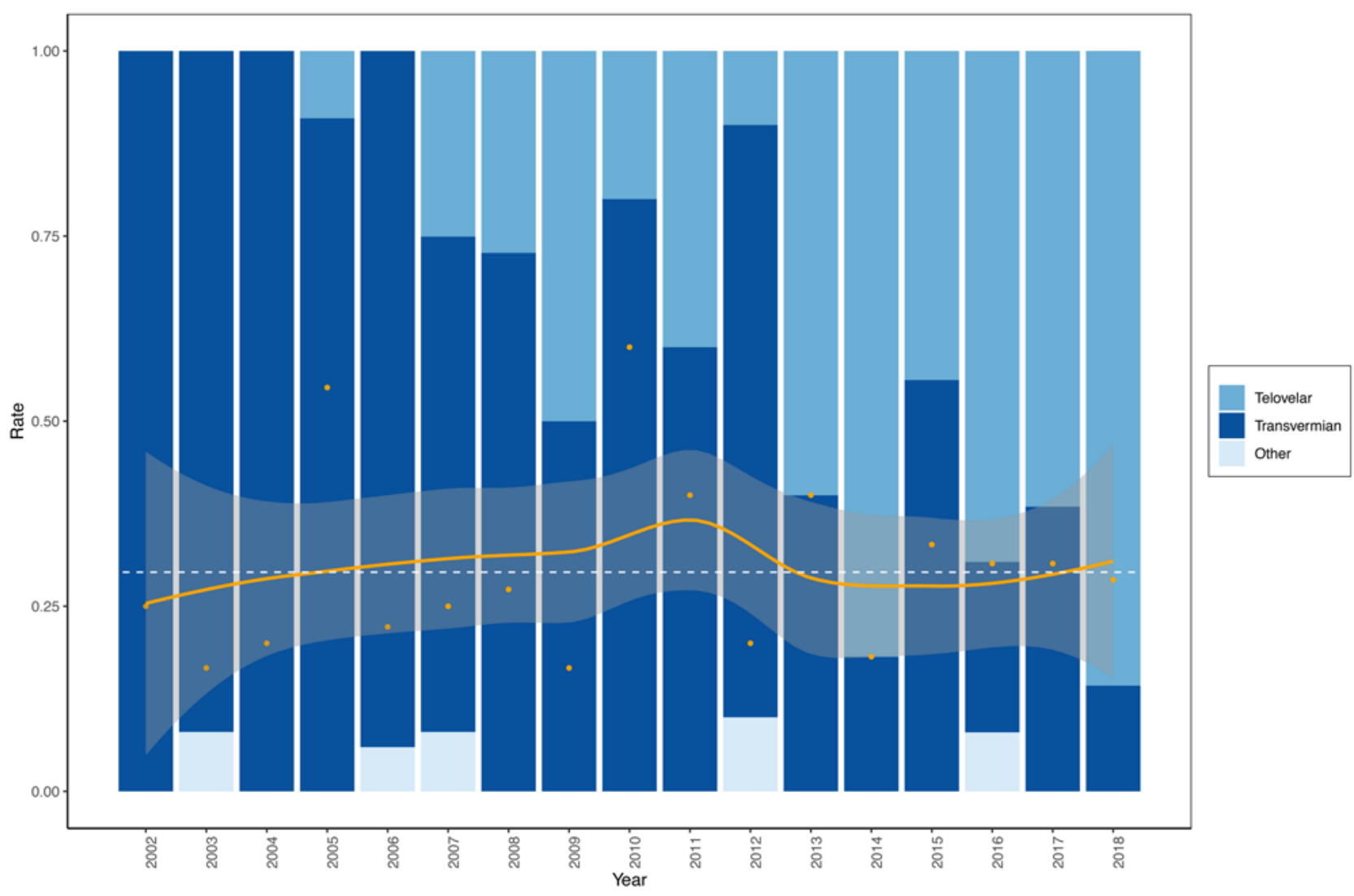

FIG. 3. Bar plot of CMS rate and rate of usage of different surgical approaches across the current cohort. The yearly CMS rate trend was plotted in a solid line using a locally weighted regression method, locally estimated scatterplot smoothing (Loess method), with error bars (standard error of proportion) shown in gray. The dashed line shows the series-wide average CMS rate. 
TABLE 3. Association of surgical approach and postoperative neurological morbidity

\begin{tabular}{lcccc}
\hline \multicolumn{1}{c}{ Characteristic } & Telovelar & Transvermian & Difference $(95 \% \mathrm{Cl})$ & p Value \\
\hline Mean tumor volume, $\mathrm{cm}^{3}$ & 31.72 & 39.13 & $7.41(5.94,8.88)$ & 0.0766 \\
\hline CMS & $17 / 55(31.0 \%)$ & $29 / 107(27.1 \%)$ & $3.8 \%(-10.2,18.9)$ & 0.745 \\
\hline Cranial neuropathy & $11 / 55(20.0 \%)$ & $19 / 107(17.8 \%)$ & $2.2 \%(-9.6,16.0)$ & 0.893 \\
\hline Diplopia & $7 / 55(12.7 \%)$ & $7 / 107(6.5 \%)$ & $6.2 \%(-2.8,18.0)$ & 0.302 \\
\hline Gait abnormality & $10 / 55(18.2 \%)$ & $11 / 107(10.3 \%)$ & $7.9 \%(-2.9,20.8)$ & 0.242 \\
\hline Sensory deficit & $2 / 55(3.6 \%)$ & $2 / 107(1.9 \%)$ & $1.8 \%(-3.6,10.6)$ & 0.605 \\
\hline Weakness $^{*}$ & $18 / 55(32.7 \%)$ & $21 / 107(19.6 \%)$ & $13.1 \%(-0.7,27.8)$ & 0.0984 \\
\hline
\end{tabular}

* One patient developed weakness after another surgical approach. Bonferroni significance level 0.05/7 $=0.00714$.

worsened weakness postoperatively. As the second most commonly encountered neurological sequela of fourth ventricle tumor surgery, this appeared to be more frequent following telovelar surgery than transvermian surgery, although this result was not statistically significant after Bonferroni multiple comparison correction (difference in proportions $13.1 \%, 95 \% \mathrm{CI}-0.7 \%$ to $27.8 \%, \mathrm{p}=0.0984)$. New or worsened cranial neuropathy occurred in 30/167 patients (18.0\%). There was no significant difference in its incidence between the telovelar and transvermian groups $(p=0.893)$. New gait abnormality and diplopia were the next most common neurological sequelae seen, at $12.6 \%$ and $8.38 \%$, respectively, and there were no significant differences in their incidence between the telovelar and transvermian groups $(p=0.242$ and 0.302 , respectively). New sensory deficit postoperatively was rare, occurring in just 4/167 patients $(2.40 \%)$.

Overall, 21/167 patients (12.6\%) developed an infective complication during their early postoperative course. There were 7 surgical site infections; 3 incidences of CSF infection (2 Staphylococcus aureus, 1 Enterococcus spp.); 4 lower respiratory tract infections; 4 urinary tract infections; 1 gastrointestinal infection; and 2 device infections (1 percutaneous endoscopic gastrostomy site, 1 tunneled vascular line).

CSF leaks occurred postoperatively in 7 cases (4.19\%). Two of these underwent wound debridement and resuturing; 2 had a lumbar puncture performed alongside resuturing; 1 had a temporary external lumbar drain placed; in 1 , the associated pseudomeningocele was tapped; and 1 was managed conservatively with oral antibiotics. There were 5 incidences of postoperative hemorrhage in this series (2.99\%), none of which required evacuation.

There were 4 deaths $(2.40 \%)$ within 30 days of operation in the patient cohort. Two patients presented in extremis, in coma or cardiorespiratory arrest. One was an 8-year-old girl who had presented with hemorrhage into a medulloblastoma, whose pupils were fixed and dilated preoperatively. Another was a 2-year-old boy who, despite resection of an anaplastic ependymoma, suffered posterior circulation infarction and hypoxic-ischemic midbrain injury during cardiorespiratory arrest at presentation preceding surgery. Two patients with metastatic medulloblastoma developed early hydrocephalus following resective surgery and, in view of their extensive disease burden, were subsequently managed palliatively.

\section{Missing Data}

In the set of 18 patients who were excluded due to missing data on surgical approach, the mean age was 5.40 years (SD 4.08 years), and this was not statistically significantly different from the mean age in the included cohort described above $(p=0.572)$. Thirteen patients were female. Overall, 5 cases of CMS (27.8\%) were seen in this cohort, which was almost identical to the proportion seen in the included cohort described above. The distribution of tumor histology seen in this group spanned the same range as that of the included cohort (5 medulloblastoma, 4 pilocytic astrocytoma, 7 ependymoma, 2 choroid plexus papilloma). There was a bimodal distribution of year of presentation in this set of patients, with 5 cases being excluded from 2005 and 3 from 2014; however, there were no other patterns seen in the missing set of data. Therefore, these were assumed to be missing completely at random, ${ }^{16}$ thus justifying the use of listwise deletion.

\section{Discussion}

This retrospective single-institution series demonstrates that resection of fourth ventricle tumors in children carries a high risk of neurological morbidity in the early postoperative period. One hundred sixty-seven patients were included in this analysis, and this comprises the largest cohort of fourth ventricle tumors, either adult or pediatric, reported in the literature. Surgical approach evolved significantly over the course of the series, with more emphasis on prone positioning and the telovelar approach. Only 49/167 patients studied across a recent 17 -year time span had no documented complications. The most common complication of fourth ventricle tumor surgery remains CMS; its incidence has remained relatively constant throughout the series ( $28.7 \%$ overall), and we found no evidence that this is influenced by favoring a telovelar over a transvermian intradural approach.

Presenting symptoms in this series were most commonly found to be vomiting and ataxia, which is in keeping with other pediatric fourth ventricle tumor series. ${ }^{4-6}$ Younger patients were more likely to present with ataxia, which is plausible because older children have betterdeveloped cerebellar motor function. Older patients were more likely to describe visual changes and headache on presentation, owing to greater language capacity. The clustering analysis revealed that children tended to present with either "global" neurological deficits (such as hydro- 


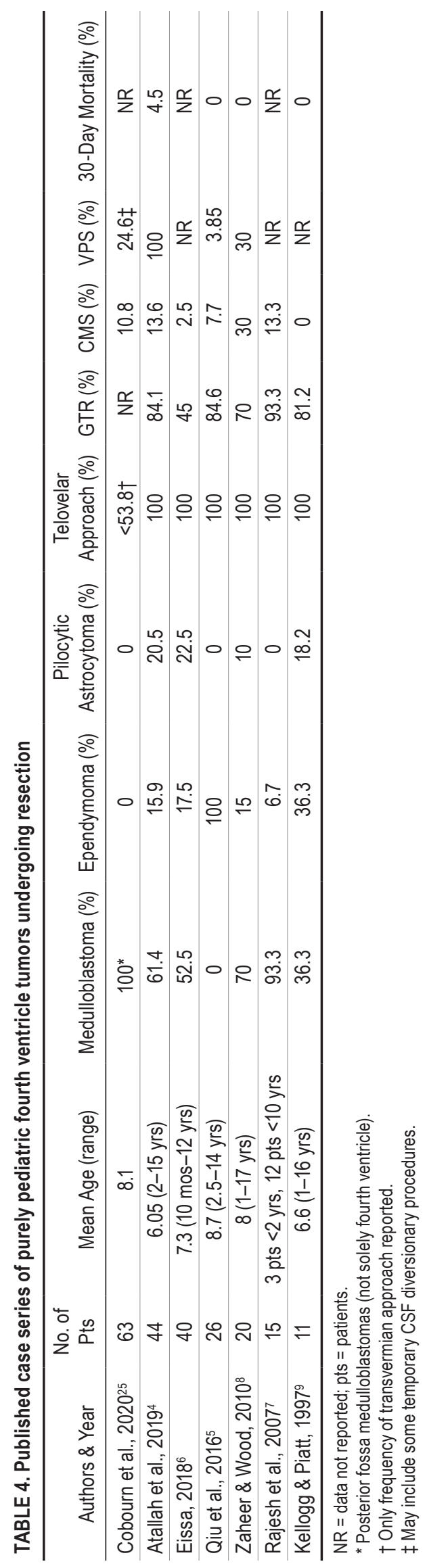

cephalus or symptoms thereof) or more focal ones (such as cranial neuropathy). In the 112 patients (67.1\%) who presented with hydrocephalus, 44 underwent EVD, and this group had a significantly higher rate of undergoing VPS insertion at a later date $(\mathrm{p}=0.00789)$. This finding (which has been previously reported ${ }^{11}$ ) is not unexpected; patients undergoing EVD were thought to have been at a higher risk of raised intracranial pressure. However, almost twothirds of patients with EVDs did not go on to receive a VPS (29/44, 65.9\%), suggesting that the hydrocephalus often resolves once tumor resection is completed. Twenty-one patients underwent ETV, of whom 8 later needed a VPS. The proportion of patients receiving shunts was higher compared with those who had not had a prior ETV (24/144, 16.7\%; difference in proportions $21.4 \%, 95 \%$ CI $2.7 \%-43.1 \%, \mathrm{p}=0.0429$ ), although this did not reach a level of statistical significance after multiple comparison correction. These findings are similar to those previously reported ${ }^{17}$ and indicate the need for careful patient selection for preresection ETV.

Overall, 33/167 patients (19.8\%) underwent VPS insertion in this series. There is a wide variation in this figure in posterior fossa tumor series reported in the literature, from $10 \%{ }^{18,19}$ to $100 \%{ }^{4}$ many lie in the range of $20 \%-30 \%{ }^{8,10-12}$

\section{Surgical Techniques}

The telovelar approach takes advantage of the cerebellomedullary fissure to arrive at and divide the membranous, noneloquent tela choroidea and inferior medullary velum ${ }^{20}$ between the lateral surface of the uvula and the medial surface of the tonsil. In so doing, the fourth ventricle can be accessed without any transgression of neural tissue. Since the first surgical case series describing its use in 11 pediatric fourth ventricle tumors, ${ }^{9}$ several more have been published in pediatric, ${ }^{4-8}$ adult,,$^{12}$ or mixed ${ }^{10,21}$ cohorts. Table 4 shows an exploration of common features across the published pediatric series.

Very large fourth ventricle tumors tend to compress the vermis. In these situations, a transvermian approach through an attenuated vermis is more straightforward than a bilateral telovelar approach. This is reflected in our results, which show a larger mean tumor volume for transvermian compared with telovelar approaches. The telovelar approach allows early identification of the floor of the fourth ventricle, early control of large tumor blood vessels arising from the posterior inferior cerebellar artery, and excellent exposure of the lateral recesses of the fourth ventricle. Although the entire fourth ventricle can be exposed through the open cerebellomedullary fissure, tumor resection in the rostral third of the fourth ventricle may be challenging; in a study using image guidance to compare transvermian and telovelar approaches, the rostral half of the fourth ventricle was more easily accessed via a transvermian approach. ${ }^{22}$

Prevailing opinion favors the telovelar approach over the transvermian route; it has been postulated that the former technique could reduce the incidence of postoperative CMS. Our findings do not support this hypothesis, and indeed there is increasing evidence suggesting that there are factors in addition to surgical approach that may be responsible for CMS. Ultimately, a randomized trial of surgical approach for fourth ventricle tumors is required 
to add definitive, high-quality evidence. However, this is not a matter in which neurosurgeons will have clinical equipoise, and decisions regarding surgical approach will probably continue to be made on an individualized basis depending on the size and rostrocaudal position of the tumor in the fourth ventricle, as well as the intent of resection and the experience of the surgeon. Anatomical particularities of such tumors may even mandate the use of both approaches simultaneously.

\section{Cerebellar Mutism Syndrome}

The overall incidence of CMS in this series was $28.7 \%$. This is broadly similar to that reported in several studies over the past 20 years, ${ }^{23,24}$ although there is some evidence that CMS is declining in incidence. ${ }^{25,26}$ Because definitions differ across published series, it is important to exercise caution in comparing incidence. There is a clear need for standardized diagnostic criteria for CMS phenomenology and severity. Currently, there are only a few published scales in use, ${ }^{27,28}$ mostly lacking clinical validation. In this study, we took a pragmatic approach to defining CMS based on review of contemporaneous medical notes by looking for the key criterion of mutism, although this probably included children with reduced speech output postoperatively, which is an inherently broad and heterogeneous group. As the field develops, the constellation of symptoms and signs that can be ascribed to CMS will become more nuanced-and certainly nonbinary-than previously thought. ${ }^{29,30}$

The neuroanatomical basis of CMS is thought to lie in the efferent cerebellar pathway, ${ }^{31,32}$ and the insult to this neural circuitry putatively arises at the time of surgery. Therefore, sparing surgical damage to cerebellar parenchyma might be expected to reduce the incidence or severity of CMS. In a series of 16 patients in whom the telovelar approach was used, none developed $\mathrm{CMS},{ }^{21}$ although this cohort was mostly composed of adult patients, in whom CMS is not a typical postoperative finding. Other series have shown that the transvermian approach appears to be a risk factor for CMS. ${ }^{33,34} \mathrm{~A}$ recent series of 63 medulloblastomas (not solely confined to the fourth ventricle) found that vermian incision "bore a statistically significant relationship to CMS," despite the simultaneous finding that a transvermian approach did not lead to a significantly higher rate of CMS. ${ }^{25}$

One key finding in this study, namely that the adoption of a telovelar approach does not significantly reduce the incidence of CMS, is corroborated by the work of other groups..$^{8,35}$ Despite this, there has been a popularization of the use of the telovelar approach in posterior fossa tumor surgery, as reflected in the literature and in the results presented here, shown graphically in Fig. 3. The latter shows that CMS rates have remained relatively constant despite an increasing proportion, year on year, of fourth ventricle tumor resections carried out via the telovelar approach, and this reflects a granularity of data on this issue that has not been reported before.

The persistence of CMS despite the increasing use of the telovelar approach in this series may not be related simply to the surgical approach itself, but rather to secondary insults, such as fixed retractor systems ${ }^{25}$ and ultrasonic aspirator use, ${ }^{36}$ however, neither of these factors was found to be significant in our analysis.

The data presented in this study suggest that the use of intraoperative ultrasonography significantly reduces the incidence of CMS ( $p=0.0365)$. Depending on the operating surgeon and the configuration of the tumor, ultrasonography was used to plan the ideal location of the cerebellar corticotomy-and therefore minimize disruption of the superficial and deep cerebellar tissue - and to assess depth of resection within the ventricle and its margins, ensuring that no uninvolved tissue, particularly at the cerebellar peduncles, was resected. Navigation by anatomical landmarks was generally considered sufficient for fourth ventricle tumors across this series-hence the infrequent use of neuronavigation software.

\section{Other Postoperative Complications}

A single-center series of fourth ventricle tumors in adults and children ${ }^{11}$ reported that $76 \%$ of patients had a documented postoperative complication. Moreover, the deficits noted (motor and gait disturbance, speech and swallowing disturbance, cranial nerve deficit, and visual impairment) are similar in frequency to those presented here. An international retrospective series of midlinethough not solely fourth ventricle-pediatric posterior fossa tumors found that the most common postoperative neurological deficit was bulbar cranial neuropathies, at $14.1 \%{ }^{37}$ (18.0\% in the current series). Allowing for differences in data collection and classification of deficits, these figures are similar to those presented in this series.

Our data indicate the low incidence of postoperative neurosurgical complications requiring reoperation, such as hemorrhage $(2.99 \%)$, CSF leak (4.19\%), and CSF infection $(1.79 \%)$. There were no incidences of postoperative stroke seen in this patient cohort. The 30-day mortality rate was $2.40 \%$, although 2 of these 4 children had already suffered irreversible neurological damage prior to surgery due to the acute nature of their presentations.

It is important to recognize that the potential complications following resective tumor surgery in the fourth ventricle are not trivial. Taken together, these figures can help provide realistic expectations to clinicians, relatives, and patients ahead of such surgery, and can be usefully quoted during the consent process.

\section{Limitations}

Our study was retrospective in nature and therefore is subject to the usual caveats of incomplete data and recall bias. The use of listwise deletion to remove subjects with incomplete data sets assumes that data are missing completely at random, ${ }^{16}$ and although this assumption is thought to be correct, it cannot be tested. Multiple imputation would have been unsuitable to address the missing data, given that most variables collected were binary. Despite our best efforts at identifying all cases of fourth ventricle tumors treated at our institution to report on the largest series of fourth ventricle tumors in the literature, the numbers in this series remain small, and results may have been affected by the missing cases. Furthermore, this is a heterogeneous group in which a total of 10 consultant neurosurgeons performed tumor resections. Although data 
collection forms were prespecified, there may have been a number of unobserved confounding factors that make it difficult to make a causal association. Finally, the focus of this communication was on the early postsurgical phase in order to faithfully describe the range of patient complications during their index neurosurgical admission, without possible confounding by subsequent chemo- or radiotherapy regimens; longer-term outcomes were therefore not part of this study.

\section{Conclusions}

In this series of pediatric fourth ventricle tumors we focused on the early postoperative phase and have shown that the resection of these tumors carries a high degree of morbidity. CMS remains the most common neurological sequela of surgery in this cohort. The incidence of CMS and other postoperative complications does not appear to be associated with the intradural surgical approach to the fourth ventricle. Open discussion of the risks surrounding fourth ventricle tumor resection in children is necessary in order to inform family and clinician expectations following surgery.

\section{Acknowledgments}

Mr. Toescu is funded by Great Ormond Street Hospital Children's Charity (award no. 174385) and is an Honorary Research Fellow of the Royal College of Surgeons of England. All research at the Great Ormond Street Hospital NHS Foundation Trust and UCL Great Ormond Street Institute of Child Health is made possible by the NIHR Great Ormond Street Hospital Biomedical Research Centre. tions.

We thank Owen Parsons for assistance with data visualiza-

\section{References}

1. Cohen-Gadol AA, Spencer DD. Inauguration of pediatric neurosurgery by Harvey W. Cushing: his contributions to the surgery of posterior fossa tumors in children. Historical vignette. J Neurosurg. 2004;100(2)(Suppl Pediatrics):225-231.

2. Matsushima T, Fukui M, Inoue T, et al. Microsurgical and magnetic resonance imaging anatomy of the cerebellomedullary fissure and its application during fourth ventricle surgery. Neurosurgery. 1992;30(3):325-330.

3. Dailey AT, McKhann GM II, Berger MS. The pathophysiology of oral pharyngeal apraxia and mutism following posterior fossa tumor resection in children. J Neurosurg. 1995;83(3): 467-475.

4. Atallah A, Rady MR, Kamal HM, et al. Telovelar approach to pediatric fourth ventricle tumors: feasibility and outcome. Turk Neurosurg. 2019;29(4):497-505.

5. Qiu BO, Wang Y, Wang W, et al. Microsurgical management of pediatric ependymomas of the fourth ventricle via the trans-cerebellomedullary fissure approach: a review of 26 cases. Oncol Lett. 2016;11(6):4099-4106.

6. Eissa EM. The role of the telovelar approach in fourth ventricular surgery: a new perspective. Turk Neurosurg. 2018; 28(4):523-529.

7. Rajesh BJ, Rao BRM, Menon G, et al. Telovelar approach: technical issues for large fourth ventricle tumors. Childs Nerv Syst. 2007;23(5):555-558.

8. Zaheer SN, Wood M. Experiences with the telovelar approach to fourth ventricular tumors in children. Pediatr Neurosurg. 2010;46(5):340-343.

9. Kellogg JX, Piatt JH Jr. Resection of fourth ventricle tumors without splitting the vermis: the cerebellomedullary fissure approach. Pediatr Neurosurg. 1997;27(1):28-33.

10. Tomasello F, Conti A, Cardali S, et al. Telovelar approach to fourth ventricle tumors: highlights and limitations. World Neurosurg. 2015;83(6):1141-1147.

11. Ferguson SD, Levine NB, Suki D, et al. The surgical treatment of tumors of the fourth ventricle: a single-institution experience. J Neurosurg. 2018;128(2):339-351.

12. Winkler EA, Birk H, Safaee M, et al. Surgical resection of fourth ventricular ependymomas: case series and technical nuances. J Neurooncol. 2016;130(2):341-349.

13. Kothari RU, Brott T, Broderick JP, et al. The ABCs of measuring intracerebral hemorrhage volumes. Stroke. 1996;27(8): 1304-1305.

14. R: A language and environment for statistical computing. 2017. Accessed July 22, 2020. https://www.r-project.org/

15. Wickham H. ggplot2: Elegant Graphics for Data Analysis. Springer; 2009.

16. Little RJA, Rubin DB. Statistical Analysis with Missing Data. 2nd ed. Wiley; 2002.

17. Srinivasan HL, Foster MT, van Baarsen K, et al. Does preresection endoscopic third ventriculostomy prevent the need for post-resection CSF diversion after pediatric posterior fossa tumor excision? A historical cohort study and review of the literature. J Neurosurg Pediatr. 2020;25(6):615-624.

18. Won S-Y, Gessler F, Dubinski D, et al. A novel grading system for the prediction of the need for cerebrospinal fluid drainage following posterior fossa tumor surgery. J Neurosurg. 2019;132(1):296-305.

19. Rappaport ZH, Shalit MN. Perioperative external ventricular drainage in obstructive hydrocephalus secondary to infratentorial brain tumours. Acta Neurochir (Wien). 1989;96(3-4): $118-121$.

20. Mussi ACM, Rhoton AL Jr. Telovelar approach to the fourth ventricle: microsurgical anatomy. J Neurosurg. 2000;92(5): 812-823.

21. El-Bahy K. Telovelar approach to the fourth ventricle: operative findings and results in 16 cases. Acta Neurochir (Wien). 2005;147(2):137-142.

22. Tanriover N, Ulm AJ, Rhoton AL Jr, Yasuda A. Comparison of the transvermian and telovelar approaches to the fourth ventricle. J Neurosurg. 2004;101(3):484-498.

23. Catsman-Berrevoets CE, Van Dongen HRH, Mulder PG, et al. Tumour type and size are high risk factors for the syndrome of "cerebellar" mutism and subsequent dysarthria. $J$ Neurol Neurosurg Psychiatry. 1999;67(6):755-757.

24. Wells EM, Khademian ZP, Walsh KS, et al. Postoperative cerebellar mutism syndrome following treatment of medulloblastoma: neuroradiographic features and origin. J Neurosurg Pediatr. 2010;5(4):329-334.

25. Cobourn K, Marayati F, Tsering D, et al. Cerebellar mutism syndrome: current approaches to minimize risk for CMS. Childs Nerv Syst. 2020;36(6):1171-1179.

26. Molinari E, Pizer B, Catsman-Berrevoets C, et al. Posterior Fossa Society Consensus Meeting 2018: a synopsis. Childs Nerv Syst. 2020;36(6):1145-1151.

27. Robertson PL, Muraszko KM, Holmes EJ, et al. Incidence and severity of postoperative cerebellar mutism syndrome in children with medulloblastoma: a prospective study by the Children's Oncology Group. J Neurosurg. 2006;105(6)(suppl): 444-451.

28. Wickenhauser ME, Khan RB, Raches D, et al. Characterizing posterior fossa syndrome: a survey of experts. Pediatr Neurol. 2020;104:19-22.

29. Schmahmann JD. Pediatric post-operative cerebellar mutism syndrome, cerebellar cognitive affective syndrome, and posterior fossa syndrome: historical review and proposed resolution to guide future study. Childs Nerv Syst. 2020;36(6): 1205-1214. 
30. Schmahmann JD. Neuroanatomy of pediatric postoperative cerebellar cognitive affective syndrome and mutism. Neurology. 2019;93(16):693-694. Published correction in Neurology. 2020;94(9):414.

31. Morris EB, Phillips NS, Laningham FH, et al. Proximal dentatothalamocortical tract involvement in posterior fossa syndrome. Brain. 2009;132(Pt 11):3087-3095.

32. Avula S. Radiology of post-operative paediatric cerebellar mutism syndrome. Childs Nerv Syst. 2020;36(6):1187-1195.

33. Grill J, Viguier D, Kieffer V, et al. Critical risk factors for intellectual impairment in children with posterior fossa tumors: the role of cerebellar damage. J Neurosurg. 2004;101(2) (suppl):152-158.

34. Kotil K, Eras M, Akçetin M, Bilge T. Cerebellar mutism following posterior fossa tumor resection in children. Turk Neurosurg. 2008;18(1):89-94.

35. Siffert J, Poussaint TY, Goumnerova LC, et al. Neurological dysfunction associated with postoperative cerebellar mutism. J Neurooncol. 2000;48(1):75-81.

36. Avula S, Mallucci C, Kumar R, Pizer B. Posterior fossa syndrome following brain tumour resection: review of pathophysiology and a new hypothesis on its pathogenesis. Childs Nerv Syst. 2015;31(10):1859-1867.

37. Renne B, Radic J, Agrawal D, et al. Cerebellar mutism after posterior fossa tumor resection in children: a multicenter international retrospective study to determine possible modifiable factors. Childs Nerv Syst. 2020;36(6):1159-1169.

\section{Disclosures}

The authors report no conflict of interest concerning the materials or methods used in this study or the findings specified in this paper.

\section{Author Contributions}

Conception and design: Aquilina, Toescu. Acquisition of data: Toescu, Samarth, Layard Horsfall, Issitt, Phipps. Analysis and interpretation of data: Aquilina, Toescu, Issitt, Margetts. Drafting the article: Aquilina, Toescu. Critically revising the article: Aquilina, Toescu, Samarth, Layard Horsfall, Issitt, Phipps, Jeelani, Thompson. Reviewed submitted version of manuscript: all authors. Approved the final version of the manuscript on behalf of all authors: Aquilina. Statistical analysis: Toescu, Issitt, Margetts. Administrative/technical/material support: Aquilina, Issitt, Margetts, Phipps, Jeelani, Thompson. Study supervision: Aquilina, Jeelani, Thompson.

\section{Supplemental Information}

\section{Previous Presentations}

Portions of this work were presented in abstract form as a poster at the Society of British Neurosurgeons Conference, London, UK, September 2018; and as an oral presentation to the International Society of Paediatric Neurosurgeons Conference, Birmingham, UK, October 2019.

\section{Correspondence}

Kristian Aquilina: Great Ormond Street Hospital for Children, London, United Kingdom. kristian.aquilina@gosh.nhs.uk. 